\title{
Targeting RV failure and cardiomyocyte injury in acute pulmonary thromboembolism
}

Jose E. Tanus-Santos

Marc Simon has reviewed the fundamental concepts underlying the diagnosis and therapy of a variety of clinical conditions associated with right ventricular (RV) failure (Simon, M. A. Assessment and treatment of right ventricular failure. Nat. Rev. Cardiol. 10, 204-218 [2013]). ${ }^{1}$ Although this Review is very clear and comprehensive, I would like to add a new perspective to the understanding of RV failure, and the therapeutic approach to this syndrome in the particular scenario of acute pulmonary thromboembolism (APT).

Multifocal necroinflammatory alterations (which reflect cell injury) are found in the right ventricle of patients with massive APT, and have major implications for both management and long-term prognosis. ${ }^{2}$ These $\mathrm{RV}$ alterations can be the result of matrix metalloproteinase (MMP) activation and release from infiltrating leucocytes or macrophages, and increase with the severity of the disease. Such changes to the right ventricle are associated with proportional increases in cardiac troponin I, a major biomarker of cardiomyocyte injury. ${ }^{3}$

MMP activation can be prevented by the use of MMP inhibitors, including the widely used antibiotic doxycycline. ${ }^{4}$ This intervention has been shown to protect against experimental APT-induced cardiomyocyte injury. ${ }^{5}$ Although doxycycline slightly attenuates RV overload as a result of decreased pulmonary vascular resistance associated with APT, ${ }^{6}$ evidence now exists that this drug also decreases mortality and $\mathrm{RV}$ deformation associated with experimental APT. ${ }^{7}$ Moreover, because oxidative stress is a major MMP-activating factor, the antioxidant tempol has also shown protective effects against APT-induced MMP activation and cardiomyocyte injury, thus preventing increases in circulating cardiac troponin I levels. ${ }^{8}$

The relevance of MMP activation during APT, and the attenuation of haemodynamic changes and cardiomyocyte injury with MMP inhibitors, might justify the use of MMP inhibitors in this critical condition. Although other agents, such as atorvastatin ${ }^{9}$ and $\mathrm{L}$-arginine ${ }^{10} \mathrm{can}$ also interrupt this critical pathophysiological mechanism, clinical trials are necessary to validate experimental findings indicating that MMP inhibition is a valid pharmacological strategy in the management of RV failure associated with APT.

Department of Pharmacology, Ribeirao Preto School of Medicine, University of Sao Paulo, Avenida dos Bandeirantes, 3900, 14049-900 Ribeirao Preto, Sao Paulo, Brazil. tanus@fmrp.usp.br

Competing interests

The author declares no competing interests.

1. Simon, M. A. Assessment and treatment of right ventricular failure. Nat. Rev. Cardiol. 10, 204-218 (2013).

2. Orde, M. M., Puranik, R., Morrow, P. L. \& Duflou, J. Myocardial pathology in pulmonary thromboembolism. Heart 97, 1695-1699 (2011).

3. Uzuelli, J. A., Dias-Junior, C. A. \& Tanus-Santos, J. E. Severity dependent increases in circulating cardiac troponin I and MMP-9 concentrations after experimental acute pulmonary thromboembolism. Clin. Chim. Acta 388, 184-188 (2008).

4. Neto-Neves, E. M., Kiss, T., Muhl, D. \& Tanus-Santos, J. E. Matrix metalloproteinases as drug targets in acute pulmonary embolism. Curr. Drug Targets 14, 344-352 (2013).

5. Neto-Neves, E. M. et al. Metalloproteinase inhibition protects against cardiomyocyte injury during experimental acute pulmonary thromboembolism. Crit. Care Med. 39 , 349-356 (2011).

6. Fortuna, G. M., Figueiredo-Lopes, L., Dias-Junior, C. A., Gerlach, R. F. \& Tanus-Santos, J. E. A role for matrix metalloproteinase-9 in the haemodynamic changes following acute pulmonary embolism. Int. J. Cardiol. 114, 22-27 (2007).

7. Cau, S. B. et al. Doxycycline prevents acute pulmonary embolism-induced mortality and right ventricular deformation in rats. Cardiovasc. Drugs Ther. 27, 259-267 (2013).

8. Sousa-Santos, O. et al. Antioxidant treatment protects against matrix metalloproteinases activation and cardiomyocyte injury during acute pulmonary thromboembolism. Naunyn Schmiedebergs Arch. Pharmacol. 385, 685-696 (2012).

9. Souza-Costa, D. C. et al. Protective effects of atorvastatin in rat models of acute pulmonary embolism: involvement of matrix metalloproteinase-9. Crit. Care Med. 35 , 239-245 (2007).

10. Souza-Costa, D. C. et al. L-Arginine attenuates acute pulmonary embolism-induced oxidative stress and pulmonary hypertension. Nitric Oxide 12, 9-14 (2005). 